\title{
Heat and Mass Transfer in a Thin Liquid Film over an Unsteady Stretching Surface in the Presence of Thermosolutal Capillarity and Variable Magnetic Field
}

\author{
Yan Zhang, Min Zhang, and Shujuan Qi \\ School of Science, Beijing University of Civil Engineering and Architecture, Beijing 100044, China \\ Correspondence should be addressed to Yan Zhang; zhangbicea@sohu.com
}

Received 20 January 2016; Accepted 30 June 2016

Academic Editor: Mohamed Abd El Aziz

Copyright (c) 2016 Yan Zhang et al. This is an open access article distributed under the Creative Commons Attribution License, which permits unrestricted use, distribution, and reproduction in any medium, provided the original work is properly cited.

\begin{abstract}
The heat and mass transfer characteristics of a liquid film which contain thermosolutal capillarity and a variable magnetic field over an unsteady stretching sheet have been investigated. The governing equations for momentum, energy, and concentration are established and transformed to a set of coupled ordinary equations with the aid of similarity transformation. The analytical solutions are obtained using the double-parameter transformation perturbation expansion method. The effects of various relevant parameters such as unsteady parameter, Prandtl number, Schmidt number, thermocapillary number, and solutal capillary number on the velocity, temperature, and concentration fields are discussed and presented graphically. Results show that increasing values of thermocapillary number and solutal capillary number both lead to a decrease in the temperature and concentration fields. Furthermore, the influences of thermocapillary number on various fields are more remarkable in comparison to the solutal capillary number.
\end{abstract}

\section{Introduction}

In the recent years, researches on the flow and heat transfer of a liquid film on an unsteady stretching sheet have got more and more attentions for its wide applications. For example, during mechanical forming processes, such as polymer extrusion, melt spinning process, the process of shaping by forcing through a die, wire and fiber coating, and food stuff process, the flow of a liquid film on an unsteady stretching sheet will be met.

In 1970, Crane [1] studied the analytic solutions of twodimensional boundary layer flow due to a stretching flat elastic sheet. Munawar et al. [2] considered time-dependent flow and heat transfer over a stretching cylinder. Shehzad et al. [3] investigated thermally radiative flow with internal heat generation and magnetic field. Furthermore, many scholars discussed other effects on the flow over a stretching sheet, such as three-dimensional flow $[4,5]$, heat and mass transfer [6-9], MHD [10], first-order chemical reaction [11], non-Newtonian fluids $[12,13]$, or different possible combinations of these above effects [14-16]. All of the above studies mainly focus on infinite fluid. In fact, the flow and heat transfer of a finite film are more suitable to describe industrial engineering. The hydrodynamics of the thin liquid film over a stretching sheet were first considered by Wang [17] who reduced the unsteady Navier-Stokes equations to the coupled nonlinear ordinary differential equations by similarity transformation and solved the problem using a kind of multiple shooting method (see Roberts and Shipman [18]). Subsequently, Wang [19] obtained the analytical solutions of a liquid thin film and confirmed the validity of the homotopy analysis method. On the basis of Wang's work [17], several authors [20-28] explored finite fluid domain of both Newtonian and non-Newtonian fluids using various velocity and thermal boundary conditions. The combined effect of viscous dissipation and magnetic field on the flow and heat transfer in a liquid film over an unsteady stretching surface was presented by Abel et al. [29]. The thermocapillary effect in finite fluid domain was first discussed by Dandapat et al. [30]. Noor and Hashim [31] extended the flow problem to hydromagnetic case. 


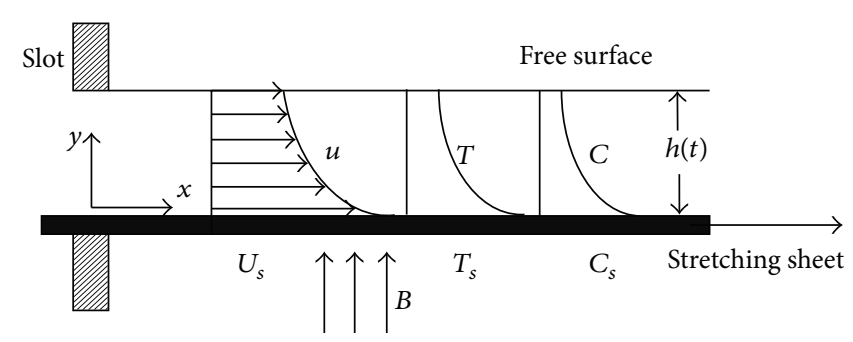

FIgURe 1: Physics model with interface condition and coordinate system.

Marangoni convection is caused by surface-tension gradient at a free liquid-gas or liquid-liquid interface that occurs due to gradient of temperature or concentration in the course of heat or mass transfer. The surface-tension variation on the free liquid surface resulting from the temperature gradient or concentration gradient can induce motion within the fluid called thermocapillary flow or solutal capillary flow (thermal Marangoni convection or solutal Marangoni convection). Pop et al. [32] investigated thermosolutal Marangoni forced convection boundary layers. On the flow field of powerlaw fluid, Lin et al. [33] analyzed the effect of radiation on Marangoni convection flow and heat transfer in the fluids with variable thermal conductivity. Then, Lin et al. [34] dealt with thermosolutal Marangoni convection flow in the presence of internal heat generation. The surface tension plays an important role on the free liquid surface. Other studies about thermocapillary effect on a thin film can be found in [35-38].

The main objective of our study is to extend previous research to the solutal Marangoni effect and mass diffusion. By means of an exact similarity transformation, governing PDEs are reduced into coupled nonlinear ODEs. And the analytical solutions are obtained using the double-parameter transformation perturbation expansion method [11]. The influences of various relevant parameters such as unsteadiness parameter $S$, Hartmann number $\mathrm{Ma}$, the Prandtl number Pr, the Schmidt number Sc, the thermocapillary number $M_{1}$, and the solutal capillary number $M_{2}$ on the flow field are elucidated through graphs and tables.

\section{Mathematical Formulation}

2.1. Governing Equations and Boundary Conditions. Consider the thin elastic sheet that emerges from a narrow slit at origin of the Cartesian coordinate system shown in Figure 1. A variable magnetic field $B=B_{0} /(1-\alpha t)^{1 / 2}$ normal to the stretching sheet is applied, where $\alpha$ is a positive constant. And in the above model, we take concentration into consideration. A thin liquid film with uniform thickness $h(t)$ rests on the horizontal sheet. By applying the boundary layer assumptions [39], the governing time-dependent equations for mass, momentum, energy, and concentration are given by

$$
\frac{\partial u}{\partial x}+\frac{\partial v}{\partial y}=0
$$

$$
\begin{aligned}
& \frac{\partial u}{\partial t}+u \frac{\partial u}{\partial x}+v \frac{\partial u}{\partial y}=v \frac{\partial^{2} u}{\partial y^{2}}-\frac{\widehat{\sigma} B^{2}}{\rho} u, \\
& \frac{\partial T}{\partial t}+u \frac{\partial T}{\partial x}+v \frac{\partial T}{\partial y}=\kappa \frac{\partial^{2} T}{\partial y^{2}}, \\
& \frac{\partial C}{\partial t}+u \frac{\partial C}{\partial x}+v \frac{\partial C}{\partial y}=D \frac{\partial^{2} C}{\partial y^{2}} .
\end{aligned}
$$

The boundary conditions are

$$
\begin{aligned}
u & =U_{s}, \\
v & =0, \\
T & =T_{s}, \\
C & =C_{s}, \\
\mu \frac{\partial u}{\partial y} & =\frac{\partial \sigma}{\partial x}, \\
\frac{\partial T}{\partial y} & =0, \\
\frac{\partial C}{\partial y} & =0, \\
v & =\frac{d h}{d t},
\end{aligned}
$$

where $u$ and $v$ are the velocity components of the fluid in the $x$-and $y$-directions, $t$ is the time, $v$ is the kinematic viscosity, $\widehat{\sigma}$ is the electrical conductivity, $B$ is the magnetic field, $\rho$ is the density, $T$ is the temperature, $\kappa$ is the thermal diffusivity, $C$ is the concentration, $D$ is the mass diffusivity, $\mu$ is dynamic viscosity, and $h(t)$ is the uniform thickness of the liquid film. The dependence of surface tension on the temperature and concentration can be expressed as [40]

$$
\sigma=\sigma_{0}\left[1-\delta_{T}\left(T-T_{0}\right)-\delta_{C}\left(C-C_{0}\right)\right],
$$

where $\delta_{T}=-\left.\left(1 / \sigma_{0}\right)(\partial \sigma / \partial T)\right|_{C}$ and $\delta_{C}=-\left.\left(1 / \sigma_{0}\right)(\partial \sigma / \partial C)\right|_{T}$. The fluid motion within liquid film resulted from not only the viscous shear arising from the stretching of the elastic sheet 
but also the surface-tension gradient. The stretching velocity $U_{s}(x, t)$ is assumed to be of the same form as that considered by Wang [17]:

$$
U_{s}=\frac{b x}{1-\alpha t}
$$

where $b$ is a positive constant and denotes the initial stretching rate. With unsteady stretching (i.e., $\alpha \neq 0$ ), however, $\alpha^{-1}$ becomes the representative time scale of the resulting unsteady boundary layer problem. The adopted formulation of the velocity sheet $U_{s}(x, t)$ in (5) is valid only for times $t<$ $\alpha^{-1}$ unless $\alpha=0$. Also the temperature and the concentration of the surface of the elastic sheet are assumed to vary both along the sheet and with time, respectively, as $[22,30]$

$$
\begin{aligned}
T_{s} & =T_{0}-T_{\text {ref }} \frac{b x^{2}}{2 v}(1-\alpha t)^{-3 / 2}, \\
C_{s} & =C_{0}-C_{\text {ref }} \frac{b x^{2}}{2 v}(1-\alpha t)^{-3 / 2},
\end{aligned}
$$

where $T_{0}$ and $C_{0}$ are the temperature and the concentration at the slit, respectively, and $T_{\text {ref }}$ and $C_{\text {ref }}$ are the reference temperature and the reference concentration in the case of $t<\alpha^{-1}$, respectively. Equation (6) represents a situation in which the sheet temperature and concentration decrease from $T_{0}$ and $C_{0}$ at the slot in proportion to $x^{2}$. The nonuniform distributions of temperature and concentration cause surface-tension gradient which leads to the fluid flow from lower surface tension to higher surface tension. According to the temperature and concentration boundary layer conditions (6), one can conclude that thermosolutal Marangoni convection flow is in accord with the flow direction of the thin film.

2.2. Similarity Transformation. The special forms of the stretching velocity, surface temperature, and fluid concentration in (5) and (6), respectively, are chosen to allow (2) to be converted into a set of ordinary differential equations by means of similarity transformations:

$$
\begin{aligned}
& u=\frac{b x}{1-\alpha t} f^{\prime}(\eta), \\
& v=-\left(\frac{v b}{1-\alpha t}\right)^{1 / 2} f(\eta) \beta, \\
& T=T_{0}-T_{\text {ref }} \frac{b x^{2}}{2 v}(1-\alpha t)^{-3 / 2} \theta(\eta), \\
& C=C_{0}-C_{\text {ref }} \frac{b x^{2}}{2 v}(1-\alpha t)^{-3 / 2} \phi(\eta),
\end{aligned}
$$

where the similarity variable $\eta$ is given by Wang [19]

$$
\eta=\left(\frac{b}{v}\right)^{1 / 2}(1-\alpha t)^{-1 / 2} y \beta^{-1}
$$

and $\beta$ is yet an unknown constant denoting the dimensionless film thickness, defined by

$$
\beta=\left(\frac{b}{v}\right)^{1 / 2}(1-\alpha t)^{-1 / 2} h(t) .
$$

Substituting (7)-(9) into (1)-(3), (1)-(3) can be transformed into the following nonlinear ordinary differential equations:

$$
\begin{array}{r}
f^{\prime \prime \prime}+\gamma\left[f f^{\prime \prime}-f^{\prime 2}-\frac{1}{2} \eta S f^{\prime \prime}-(S+\mathrm{Ma}) f^{\prime}\right]=0, \\
\operatorname{Pr}^{-1} \theta^{\prime \prime}+\gamma\left(f \theta^{\prime}-\frac{3}{2} S \theta-\frac{1}{2} S \theta^{\prime} \eta-2 f^{\prime} \theta\right)=0, \\
\mathrm{Sc}^{-1} \phi^{\prime \prime}+\gamma\left(f \phi^{\prime}-\frac{3}{2} S \phi-\frac{1}{2} S \phi^{\prime} \eta-2 f^{\prime} \phi\right)=0,
\end{array}
$$

subject to the boundary conditions

$$
\begin{aligned}
f(0) & =0, \\
f^{\prime}(0) & =1, \\
\theta(0) & =1, \\
\phi(0) & =1, \\
f(1) & =\frac{S}{2}, \\
f^{\prime \prime}(1) & =M_{1} \theta(1)+M_{2} \phi(1), \\
\theta^{\prime}(1) & =0, \\
\phi^{\prime}(1) & =0,
\end{aligned}
$$$$
\text { at } \eta=0 \text {, }
$$

$$
\text { at } \eta=1 \text {, }
$$

where Ma $=\widehat{\sigma} B_{0} /(\rho b)$ is Hartmann number, $S=\alpha / b$ is the dimensionless unsteadiness parameter, $\operatorname{Pr}=v / \kappa$ is the Prandtl number, Sc $=v / D$ is the Schmidt number, $\gamma=\beta^{2}$ is the dimensionless film thickness, and the thermocapillary number $M_{1}$ and the solutal capillary number $M_{2}$ are defined as $M_{1}=\delta_{T} \sigma_{0} T_{\text {ref }} \beta / \mu \sqrt{b v}$ and $M_{2}=\delta_{C} \sigma_{0} C_{\text {ref }} \beta / \mu \sqrt{b v}$, respectively.

The skin friction coefficient $C_{f}$ and the Nusselt number $\mathrm{Nu}_{x}$ are defined as

$$
\begin{aligned}
C_{f} & =\frac{\tau_{w}}{\rho U_{s}^{2} / 2}, \\
\mathrm{Nu}_{x} & =\frac{q_{w} x}{k T_{\mathrm{ref}}}
\end{aligned}
$$

where the wall skin friction $\tau_{w}$ and the sheet heat flux $q_{w}$ are

$$
\begin{aligned}
& \tau_{w}=\mu\left(\frac{\partial u}{\partial y}\right)_{y=0}, \\
& q_{w}=-k\left(\frac{\partial T}{\partial y}\right)_{y=0} .
\end{aligned}
$$


TABLE 1: Comparison of values of film thickness $\beta$ and skin friction coefficient $-f^{\prime \prime}(0)$ with $\mathrm{Ma}=0$.

\begin{tabular}{lcccc}
\hline \multirow{S}{*}{$S$} & \multicolumn{2}{c}{ Wang [19] } & \multicolumn{2}{c}{ Present results } \\
& $\beta$ & $-f^{\prime \prime}(0)$ & $\beta$ & $-f^{\prime \prime}(0)$ \\
\hline 0.8 & 2.151990 & 2.680940 & 2.152839 & 2.681920 \\
1.0 & 1.543620 & 1.972385 & 1.543620 & 1.972385 \\
1.2 & 1.127781 & 1.442631 & 1.127781 & 1.442625 \\
1.4 & 0.821032 & 1.012784 & 0.821032 & 1.012780 \\
\hline
\end{tabular}

Substituting (14) into (13), then we get

$$
\begin{aligned}
C_{f} & =\frac{2}{\beta} f^{\prime \prime}(0) \mathrm{Re}_{x}{ }^{-1 / 2}, \\
\mathrm{Nu}_{x} & =\frac{1}{2 \beta(1-\alpha t)} \theta^{\prime}(0) \operatorname{Re}_{x}^{3 / 2},
\end{aligned}
$$

where $\operatorname{Re}_{x}=U_{s} x / v$ is the local Reynolds number.

2.3. Solution Approach. In order to solve (10), We used the double-parameter transformation perturbation expansion method that confirmed the validity by Zhang and Zheng [11]. We introduced an artificial small parameter $\varepsilon$; the equations can be expanded to the form of power series. We can obtain analytical solutions in the form of series by comparing the coefficients of same power and solving the equation sets.

We transform the dependent variable and independent variable as follows:

$$
\begin{aligned}
f(\eta) & =\varepsilon g(\xi)+\eta+\frac{\alpha_{1}}{2} \eta^{2} \\
& =\varepsilon g(\xi)+\varepsilon^{1 / 3} \xi+\frac{\alpha_{1}}{2} \varepsilon^{2 / 3} \xi^{2} \\
\theta(\eta) & =\varepsilon^{2 / 3} h(\xi)+1+\alpha_{2} \eta=\varepsilon^{2 / 3} h(\xi)+1+\alpha_{2} \varepsilon^{1 / 3} \xi \\
\phi(\eta) & =\varepsilon^{2 / 3} \omega(\xi)+1+\alpha_{3} \eta=\varepsilon^{2 / 3} \omega(\xi)+1+\alpha_{3} \varepsilon^{1 / 3} \xi
\end{aligned}
$$

where $\xi=\varepsilon^{-1 / 3} \eta$ and $\varepsilon$ is an artificial small parameter. We assume $f^{\prime \prime}(0)=\alpha_{1}, \theta^{\prime}(0)=\alpha_{2}$, and $\phi^{\prime}(0)=\alpha_{3}$; here $\alpha_{1}, \alpha_{2}$, and $\alpha_{3}$ are constants; the boundary conditions become

$$
\begin{gathered}
g(0)=0, \\
g^{\prime}(0)=0, \\
g^{\prime \prime}(0)=0, \\
h(0)=0, \\
h^{\prime}(0)=0, \\
\omega(0)=0, \\
\omega^{\prime}(0)=0 .
\end{gathered}
$$

Equation (10) reduces to

$$
\begin{aligned}
& g^{\prime \prime \prime}(\xi)+\gamma\left(\left(\varepsilon g(\xi)+\varepsilon^{1 / 3} \xi+\frac{\alpha_{1}}{2} \varepsilon^{2 / 3} \xi^{2}\right)\left(\varepsilon^{1 / 3} g^{\prime \prime}+\alpha_{1}\right)\right. \\
& -\frac{1}{2} S \varepsilon^{1 / 3} \xi\left(\varepsilon^{1 / 3} g^{\prime \prime}+\alpha_{1}\right)-\left(\varepsilon^{2 / 3} g^{\prime}+1+\alpha_{1} \varepsilon^{1 / 3} \xi\right)^{2} \\
& \left.-(S+\mathrm{Ma})\left(\varepsilon^{2 / 3} g^{\prime}+1+\alpha_{1} \varepsilon^{1 / 3} \xi\right)\right)=0, \\
& \frac{h^{\prime \prime}(\xi)}{\operatorname{Pr}}+\gamma\left(\left(\varepsilon g_{0}(\xi)+\varepsilon^{1 / 3} \xi+\frac{\alpha_{1}}{2} \varepsilon^{2 / 3} \xi^{2}\right)\right. \\
& \cdot\left(\varepsilon^{1 / 3} h^{\prime}(\xi)+\alpha_{2}\right)-\left(2\left(\varepsilon^{2 / 3} g^{\prime}(\xi)+1+\alpha_{1} \varepsilon^{1 / 3} \xi\right)\right) \\
& \cdot\left(\varepsilon^{2 / 3} h(\xi)+1+\alpha_{2} \varepsilon^{1 / 3} \xi\right)-\frac{1}{2} \\
& \cdot S \varepsilon^{1 / 3} \xi\left(\varepsilon^{1 / 3} h^{\prime}(\xi)+\alpha_{2}\right) \\
& \left.-\frac{3 S}{2}\left(\varepsilon^{2 / 3} h(\xi)+1+\alpha_{2} \varepsilon^{1 / 3} \xi\right)\right)=0, \\
& \frac{\omega^{\prime \prime}(\xi)}{\mathrm{Sc}}+\gamma\left(\left(\varepsilon g_{0}(\xi)+\varepsilon^{1 / 3} \xi+\frac{\alpha_{1}}{2} \varepsilon^{2 / 3} \xi^{2}\right)\right. \\
& \cdot\left(\varepsilon^{1 / 3} \omega^{\prime}(\xi)+\alpha_{3}\right)-\left(2\left(\varepsilon^{2 / 3} g^{\prime}(\xi)+1+\alpha_{1} \varepsilon^{1 / 3} \xi\right)\right) \\
& \cdot\left(\varepsilon^{2 / 3} \omega(\xi)+1+\alpha_{3} \varepsilon^{1 / 3} \xi\right)-\frac{1}{2} \\
& \cdot S \varepsilon^{1 / 3} \xi\left(\varepsilon^{1 / 3} \omega^{\prime}(\xi)+\alpha_{3}\right) \\
& \left.-\frac{3 S}{2}\left(\varepsilon^{2 / 3} \omega(\xi)+1+\alpha_{3} \varepsilon^{1 / 3} \xi\right)\right)=0 .
\end{aligned}
$$

And we can assume

$$
\begin{aligned}
g(\xi)= & g_{0}(\xi)+\varepsilon^{1 / 3} g_{1}(\xi)+\varepsilon^{2 / 3} g_{2}(\xi)+\varepsilon g_{3}(\xi) \\
& +\varepsilon^{4 / 3} g_{4}(\xi)+\cdots, \\
h(\xi)= & h_{0}(\xi)+\varepsilon^{1 / 3} h_{1}(\xi)+\varepsilon^{2 / 3} h_{2}(\xi)+\varepsilon h_{3}(\xi) \\
& +\varepsilon^{4 / 3} h_{4}(\xi)+\cdots, \\
\omega(\xi)= & \omega_{0}(\xi)+\varepsilon^{1 / 3} \omega_{1}(\xi)+\varepsilon^{2 / 3} \omega_{2}(\xi)+\varepsilon \omega_{3}(\xi) \\
& +\varepsilon^{4 / 3} \omega_{4}(\xi)+\cdots
\end{aligned}
$$

with the boundary conditions

$$
\begin{aligned}
& g_{n}(0)=0, \\
& g_{n}^{\prime}(0)=0, \\
& g_{n}^{\prime \prime}(0)=0, \\
& g_{n}^{\prime \prime \prime}(0)=0,
\end{aligned}
$$$$
n=0,1,2, \ldots,
$$ 
TAble 2: Values of $\beta, f^{\prime}(1), \theta(1)$ and $\phi(1),-f^{\prime \prime}(0),-\theta^{\prime}(0)$, and $-\phi^{\prime}(0)$ with $\mathrm{Ma}=1.2, M_{1}=1, M_{2}=1, \operatorname{Pr}=1$, and $\mathrm{Sc}=1.8$ and $S$ is varied.

\begin{tabular}{lccccccc}
\hline$S$ & $\beta$ & $f^{\prime}(1)$ & $\theta(1)$ & $\phi(1)$ & $-f^{\prime \prime}(0)$ & $-\theta^{\prime}(0)$ & $-\phi^{\prime}(0)$ \\
\hline 0.8 & 1.706139 & 0.245858 & 0.190409 & 0.079435 & 2.791406 & 2.738673 & 3.835584 \\
1.0 & 1.340872 & 0.387092 & 0.249442 & 0.118379 & 2.180379 & 2.252363 & 3.160992 \\
1.2 & 1.088400 & 0.541510 & 0.309461 & 0.161276 & 1.714381 & 1.897617 & 2.678301 \\
\hline
\end{tabular}

TABle 3: Values of $\beta, f^{\prime}(1), \theta(1)$ and $\phi(1),-f^{\prime \prime}(0),-\theta^{\prime}(0)$, and $-\phi^{\prime}(0)$ with $S=0.8, M_{1}=1, M_{2}=1, \operatorname{Pr}=1$, and $\mathrm{Sc}=1.8$ and $\mathrm{Ma}$ is varied.

\begin{tabular}{cccccccc}
\hline $\mathrm{Ma}$ & $\beta$ & $f^{\prime}(1)$ & $\theta(1)$ & $\phi(1)$ & $-f^{\prime \prime}(0)$ & $-\theta^{\prime}(0)$ & $-\phi^{\prime}(0)$ \\
\hline 0.5 & 1.955885 & 0.228035 & 0.131483 & 0.047797 & 2.776933 & 3.21537 & 4.463988 \\
1.5 & 1.629020 & 0.252600 & 0.211365 & 0.092719 & 2.802801 & 2.590747 & 3.640895 \\
3.0 & 1.370781 & 0.283080 & 0.299672 & 0.154063 & 2.866680 & 2.089107 & 2.983500 \\
\hline
\end{tabular}

TABle 4: Values of $\beta, f^{\prime}(1), \theta(1)$ and $\phi(1),-f^{\prime \prime}(0),-\theta^{\prime}(0)$, and $-\phi^{\prime}(0)$ with $S=0.8, \mathrm{Ma}=1, M_{2}=1, \operatorname{Pr}=1$, and $\mathrm{Sc}=1.8$ and $M_{1}$ is varied.

\begin{tabular}{lccccccc}
\hline$M_{1}$ & $\beta$ & $f^{\prime}(1)$ & $\theta(1)$ & $\phi(1)$ & $-f^{\prime \prime}(0)$ & $-\theta^{\prime}(0)$ & $-\phi^{\prime}(0)$ \\
\hline 1 & 1.765648 & 0.242329 & 0.178412 & 0.070413 & 2.783885 & 2.850602 & 3.984435 \\
3 & 1.895449 & 0.296754 & 0.143799 & 0.054085 & 2.978572 & 3.080157 & 4.285635 \\
5 & 1.987330 & 0.335046 & 0.123865 & 0.044928 & 3.120442 & 3.24009 & 4.498705 \\
8 & 2.089998 & 0.380759 & 0.106127 & 0.036553 & 3.279882 & 3.415187 & 4.733832 \\
\hline
\end{tabular}

TABle 5: Values of $\beta, f^{\prime}(1), \theta(1)$ and $\phi(1),-f^{\prime \prime}(0),-\theta^{\prime}(0)$, and $-\phi^{\prime}(0)$ with $S=0.8, \mathrm{Ma}=1, M_{1}=1, \operatorname{Pr}=1$, and $\mathrm{Sc}=1.8$ and $M_{2}$ is varied.

\begin{tabular}{lccccccc}
\hline$M_{2}$ & $\beta$ & $f^{\prime}(1)$ & $\theta(1)$ & $\phi(1)$ & $-f^{\prime \prime}(0)$ & $-\theta^{\prime}(0)$ & $-\phi^{\prime}(0)$ \\
\hline 1 & 1.765648 & 0.242329 & 0.178412 & 0.070413 & 2.783885 & 2.850602 & 3.984435 \\
3 & 1.823807 & 0.265936 & 0.162696 & 0.062564 & 2.870952 & 2.95405 & 4.120261 \\
5 & 1.870447 & 0.284213 & 0.148046 & 0.056928 & 2.942314 & 3.03796 & 4.229606 \\
8 & 1.927131 & 0.30837 & 0.135444 & 0.050747 & 3.028503 & 3.136665 & 4.360637 \\
\hline
\end{tabular}

TABle 6: Values of $\beta, f^{\prime}(1), \theta(1)$ and $\phi(1),-f^{\prime \prime}(0),-\theta^{\prime}(0)$, and $-\phi^{\prime}(0)$ with $S=0.8, \mathrm{Ma}=1, M_{1}=1, M_{2}=1$, and $\mathrm{Sc}=1.8$ and Pr is varied.

\begin{tabular}{lccccccc}
\hline $\operatorname{Pr}$ & $\beta$ & $f^{\prime}(1)$ & $\theta(1)$ & $\phi(1)$ & $-f^{\prime \prime}(0)$ & $-\theta^{\prime}(0)$ & $-\phi^{\prime}(0)$ \\
\hline 1 & 1.765648 & 0.242329 & 0.178412 & 0.070413 & 2.783885 & 2.850602 \\
2 & 1.70801 & 0.216269 & 0.065849 & 0.079270 & 2.701304 & 4.083229 & 3.984435 \\
5 & 1.675911 & 0.203976 & 0.009869 & 0.084604 & 2.654055 & 6.569892 & 3.775763 \\
10 & 1.671722 & 0.20159 & 0.000697 & 0.085353 & 2.648593 & 9.447705 & 3.766435 \\
\hline
\end{tabular}

TABle 7: Values of $\beta, f^{\prime}(1), \theta(1)$ and $\phi(1),-f^{\prime \prime}(0),-\theta^{\prime}(0)$, and $-\phi^{\prime}(0)$ with $S=0.8, \mathrm{Ma}=1, M_{1}=1, M_{2}=1$, and $\mathrm{Pr}=1$ and Sc is varied.

\begin{tabular}{lccccccc}
\hline Sc & $\beta$ & $f^{\prime}(1)$ & $\theta(1)$ & $\phi(1)$ & $-f^{\prime \prime}(0)$ & $-\theta^{\prime}(0)$ & $-\phi^{\prime}(0)$ \\
\hline 0.5 & 1.884207 & 0.289866 & 0.144404 & 0.313734 & 2.963305 & 3.062276 & 2.011923 \\
1 & 1.810615 & 0.259498 & 0.163349 & 0.160799 & 2.852006 & 2.932347 & 2.933503 \\
1.5 & 1.77745 & 0.245319 & 0.170494 & 0.093966 & 2.80298 & 2.874491 & 3.62599 \\
1.8 & 1.765648 & 0.242329 & 0.178412 & 0.070413 & 2.783885 & 2.850602 & 3.984435 \\
\hline
\end{tabular}

$$
\begin{array}{cl}
h_{n}(0)=0, & \omega_{n}(0)=0, \\
h_{n}^{\prime}(0)=0, & \omega_{n}^{\prime}(0)=0, \\
h_{n}^{\prime \prime}(0)=0, & \omega_{n}^{\prime \prime}(0)=0, \\
n=0,1,2, \ldots, & n=0,1,2, \ldots
\end{array}
$$



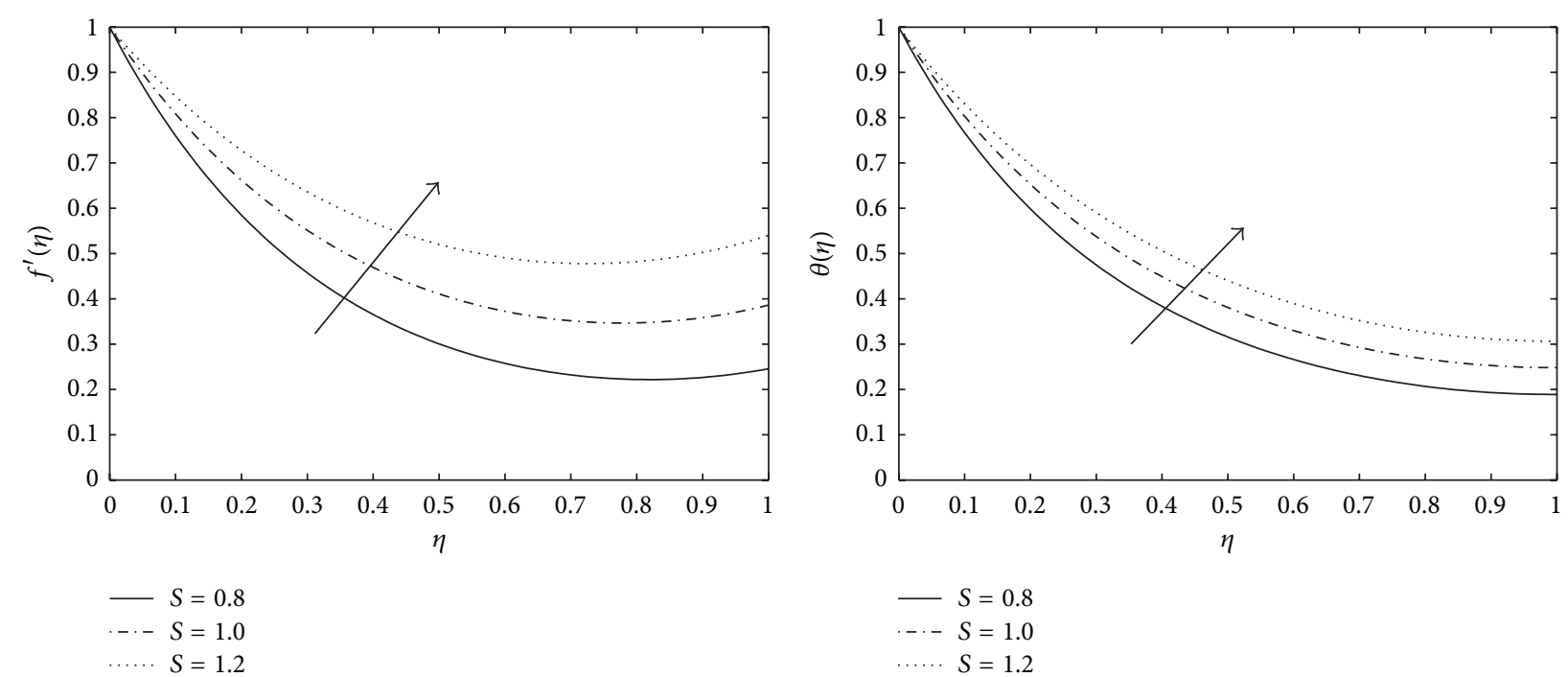

(a)

(b)

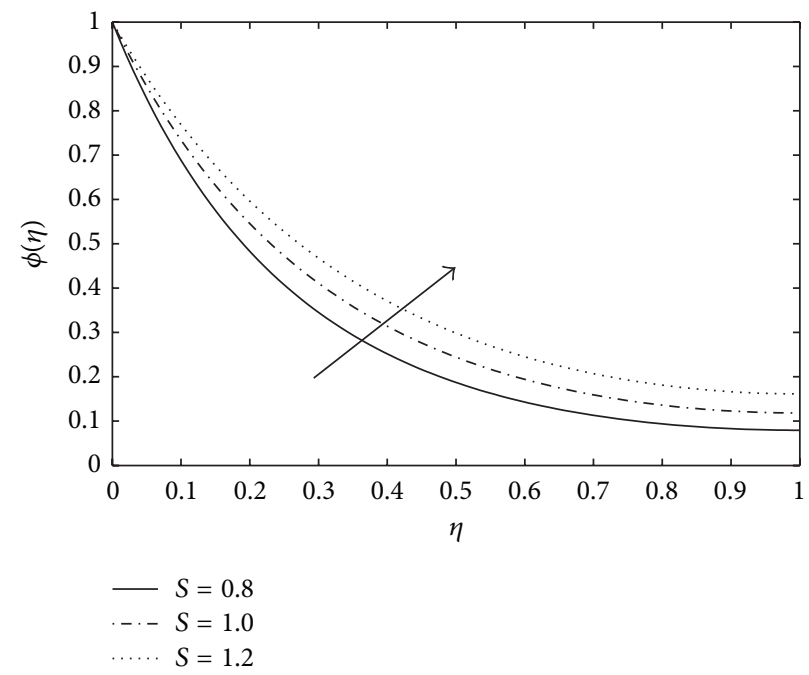

(c)

Figure 2: (a) Velocity profiles for different values of $S$ with $\mathrm{Ma}=1.2, M_{1}=1, M_{2}=1$, $\operatorname{Pr}=1$, and $\mathrm{Sc}=1.8$. (b) Temperature profiles for different values of $S$ with $\mathrm{Ma}=1.2, M_{1}=1, M_{2}=1, \operatorname{Pr}=1$, and $\mathrm{Sc}=1.8$. (c) Concentration profiles for different values of $S$ with $\mathrm{Ma}=1.2$, $M_{1}=1, M_{2}=1, \operatorname{Pr}=1$, and $\mathrm{Sc}=1.8$.

Let $S=0.8$ and $\mathrm{Ma}=1$, and we can obtain

$$
\begin{aligned}
& f(\eta)=\eta+\frac{1}{2} \alpha_{1} \eta^{2}+\frac{7}{15} \gamma \eta^{3}+\frac{2}{15} \alpha_{1} \gamma \eta^{4} \\
& +\left(\frac{1}{120} \alpha_{1}^{2} \gamma+\frac{91}{1500} \gamma^{2}\right) \eta^{5}+\frac{1}{60} \alpha_{1} \gamma^{2} \eta^{6} \\
& +\left(\frac{23}{25200} \alpha_{1}^{2} \gamma^{2}+\frac{77}{15000} \gamma^{3}\right) \eta^{7} \\
& +\left(\frac{589}{504000} \alpha_{1} \gamma^{3}-\frac{77}{40320} \alpha_{1}^{3} \gamma^{2}\right) \eta^{8} \\
& +\left(\frac{383}{9072000} \alpha_{1}^{2} \gamma^{3}+\frac{3871}{16200000} \gamma^{4}\right) \eta^{9} \\
& +\left(\frac{1}{432000} \alpha_{1}^{3} \gamma^{3}+\frac{209}{7560000} \alpha_{1} \gamma^{4}\right) \eta^{10}+\cdots
\end{aligned}
$$$$
f^{\prime}(\eta)=1+\alpha_{1} \eta+\frac{7}{45} \gamma \eta^{2}+\frac{1}{30} \alpha_{1} \gamma \eta^{3}
$$$$
+\left(\frac{1}{600} \alpha_{1}^{2} \gamma+\frac{91}{4500} \gamma^{2}\right) \eta^{4}+\frac{1}{360} \alpha_{1} \gamma^{2} \eta^{5}
$$$$
+\left(\frac{23}{176400} \alpha_{1}^{2} \gamma^{2}+\frac{11}{15000} \gamma^{3}\right) \eta^{6}
$$$$
+\left(\frac{589}{4672000} \alpha_{1} \gamma^{3}-\frac{77}{322560} \alpha_{1}^{3} \gamma^{2}\right) \eta^{7}
$$$$
+\left(\frac{383}{81648000} \alpha_{1}^{2} \gamma^{3}+\frac{3871}{145800000} \gamma^{4}\right) \eta^{8}
$$$$
+\left(\frac{1}{4320000} \alpha_{1}^{3} \gamma^{3}+\frac{209}{75600000} \alpha_{1} \gamma^{4}\right) \eta^{9}+\cdots,
$$ 


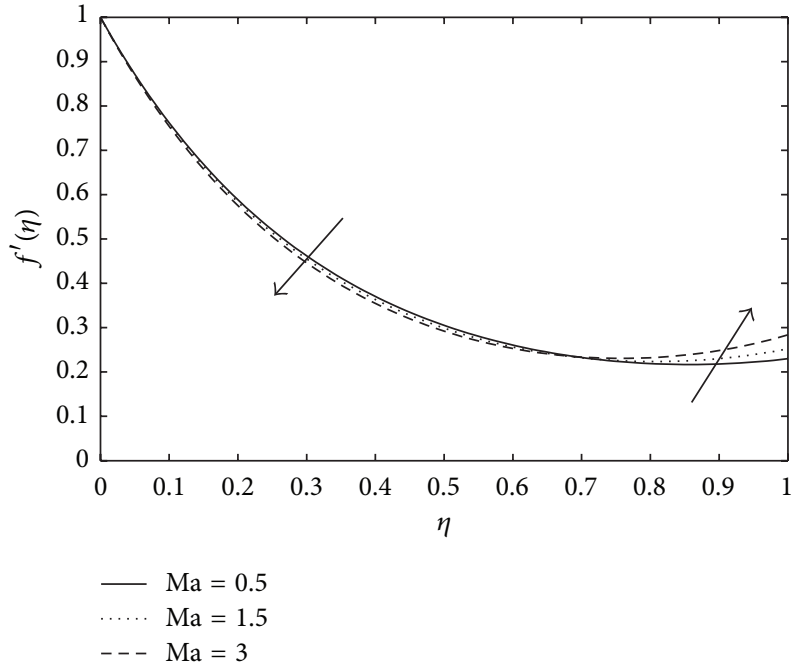

(a)

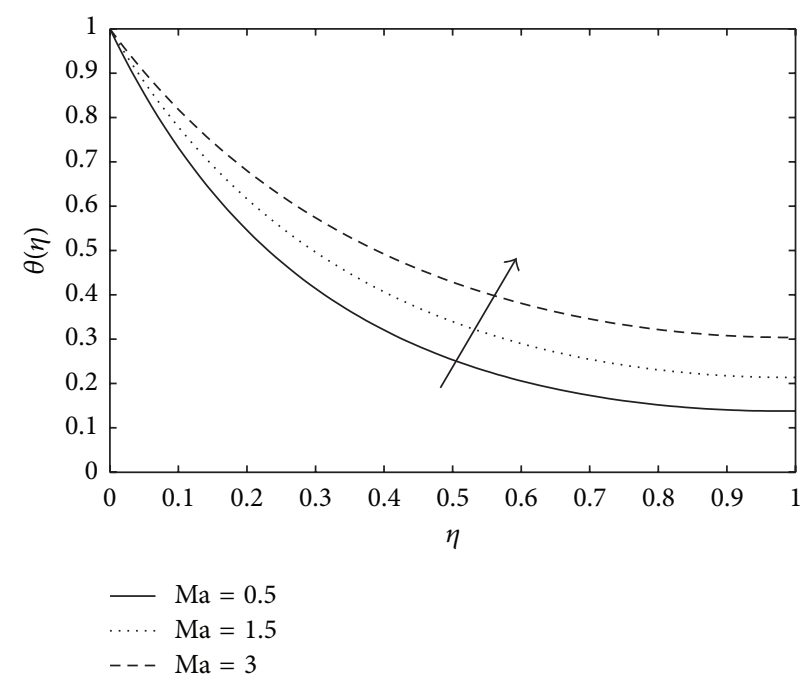

(b)

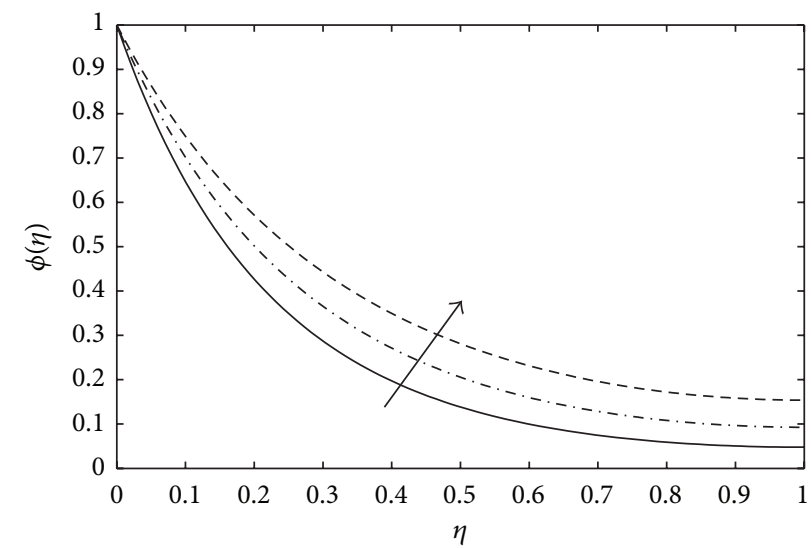

- $\mathrm{Ma}=0.5$

... $\mathrm{Ma}=1.5$

- - $\mathrm{Ma}=3$

(c)

Figure 3: (a) Velocity profiles for different values of Ma with $S=0.8, M_{1}=1, M_{2}=1, \operatorname{Pr}=1$, and $\mathrm{Sc}=1.8$. (b) Temperature profiles for different values of Ma with $S=0.8, M_{1}=1, M_{2}=1, \operatorname{Pr}=1$, and $\mathrm{Sc}=1.8$. (c) Concentration profiles for different values of Ma with $S=0.8$, $M_{1}=1, M_{2}=1, \operatorname{Pr}=1$, and $\mathrm{Sc}=1.8$.

$$
\begin{array}{lll}
f^{\prime \prime}(\eta)=\alpha_{1}+\frac{7}{90} \gamma \eta+\frac{1}{90} \alpha_{1} \gamma \eta^{2} & \theta(\eta)=\alpha_{2} \eta+\frac{8}{5} \gamma \eta^{2}+\left(\frac{13}{30} \gamma \alpha_{2}+\frac{1}{3} \alpha_{1} \gamma\right) \eta^{3} \\
+\left(\frac{1}{2400} \alpha_{1}^{2} \gamma+\frac{91}{18000} \gamma^{2}\right) \eta^{3}+\frac{1}{1800} \alpha_{1} \gamma^{2} \eta^{4} & +\left(\frac{1}{2} \gamma^{2}+\frac{1}{8} \gamma \alpha_{2} \alpha_{1}\right) \eta^{4} \\
+\left(\frac{23}{1058400} \alpha_{1}{ }^{2} \gamma^{2}+\frac{11}{90000} \gamma^{3}\right) \eta^{5} & +\left(\frac{147}{1000} \gamma^{2} \alpha_{2}+\frac{47}{300} \alpha_{1} \gamma^{2}\right) \eta^{5} \\
+\left(\frac{589}{34804000} \alpha_{1} \gamma^{3}-\frac{77}{2257920} \alpha_{1}^{3} \gamma^{2}\right) \eta^{6} & +\left(\frac{1}{120} \gamma^{2} \alpha_{1}^{2}+\frac{599}{4500} \gamma^{3}+\frac{1}{24} \gamma^{2} \alpha_{1} \alpha_{2}\right) \eta^{6} \\
+\left(\frac{383}{653184000} \alpha_{1}^{2} \gamma^{3}+\frac{3871}{116400000} \gamma^{4}\right) \eta^{7} & +\left(\frac{989}{21000} \alpha_{1} \gamma^{3}+\frac{2533}{9000} \gamma^{3} \alpha_{2}+\frac{1}{560} \gamma^{2} \alpha_{2} \alpha_{1}^{2}\right) \eta^{7} \\
+\left(\frac{1}{38880000} \alpha_{1}^{3} \gamma^{3}+\frac{209}{680400000} \alpha_{1} \gamma^{4}\right) \eta^{8}+\cdots, & +\cdots,
\end{array}
$$




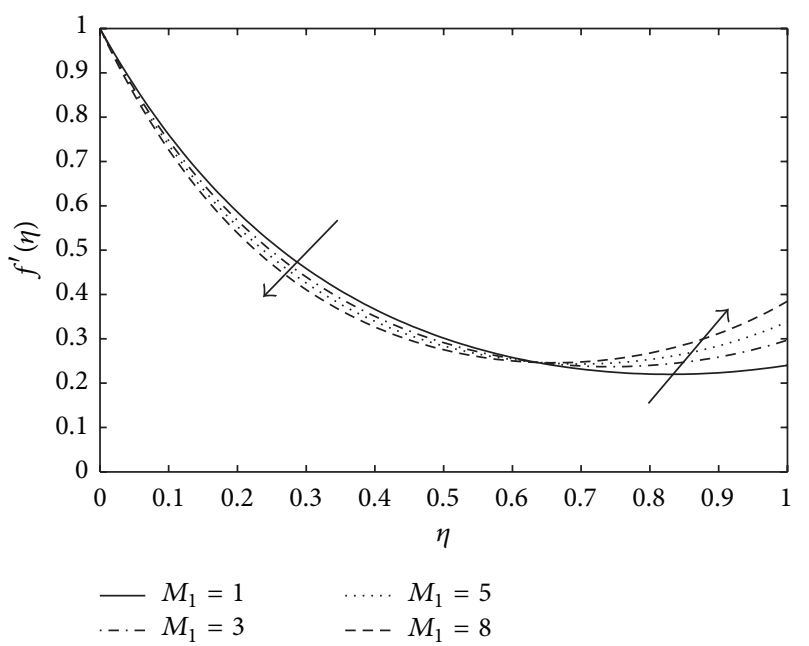

(a)

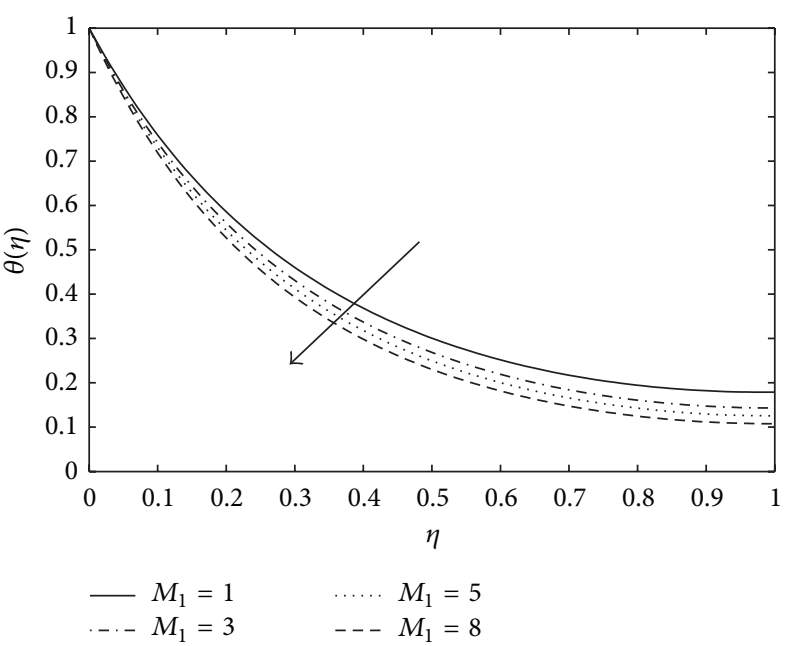

(b)

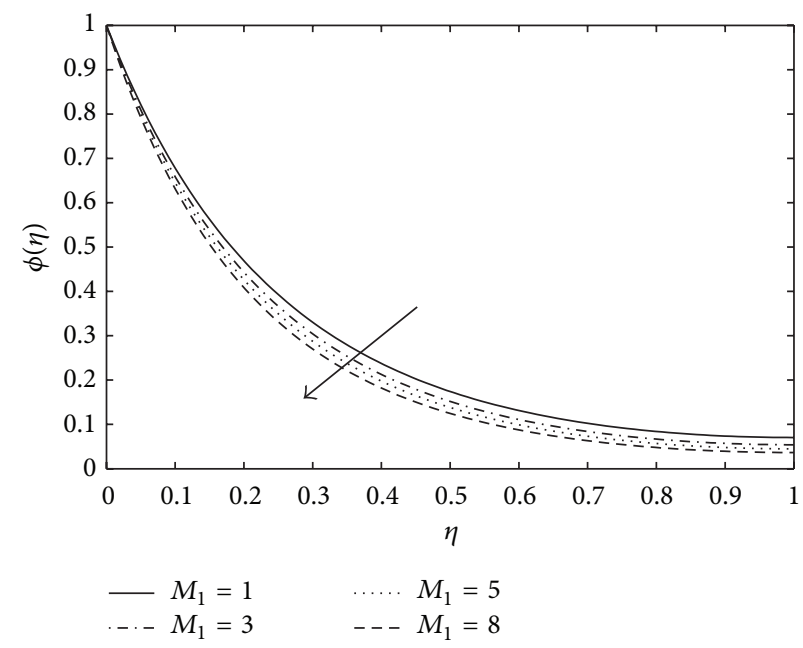

(c)

FIGURE 4: (a) Velocity profiles for different values of $M_{1}$ with $S=0.8, \mathrm{Ma}=1, M_{2}=1, \mathrm{Pr}=1$, and $\mathrm{Sc}=1.8$. (b) Temperature profiles for different values of $M_{1}$ with $S=0.8, \mathrm{Ma}=1, M_{2}=1, \operatorname{Pr}=1$, and $\mathrm{Sc}=1.8$. (c) Concentration profiles for different values of $M_{1}$ with $S=0.8$, $\mathrm{Ma}=1, M_{2}=1, \operatorname{Pr}=1$, and $\mathrm{Sc}=1.8$.

$$
\begin{array}{lll}
\theta^{\prime}(\eta)=\alpha_{2}+\frac{4}{5} \gamma \eta+\left(\frac{13}{90} \gamma \alpha_{2}+\frac{1}{9} \alpha_{1} \gamma\right) \eta^{2} & +\left(\frac{23}{15} \gamma^{2}+\frac{1}{4} \gamma \alpha_{1} \alpha_{3}\right) \eta^{4} \\
+\left(\frac{1}{8} \gamma^{2}+\frac{1}{32} \gamma \alpha_{1} \alpha_{2}\right) \eta^{3} & +\left(\frac{133}{375} \gamma^{2} \alpha_{3}+\frac{13}{25} \gamma^{2} \alpha_{1}\right) \eta^{5} \\
+\left(\frac{147}{5000} \gamma^{2} \alpha_{2}+\frac{47}{1500} \alpha_{1} \gamma^{2}\right) \eta^{4} & +\left(\frac{1171}{2250} \gamma^{3}+\frac{1}{36} \gamma^{2} \alpha_{1}^{2}+\frac{47}{450} \gamma^{2} \alpha_{1} \alpha_{3}\right) \eta^{6} \\
+\left(\frac{1}{720} \gamma^{2} \alpha_{1}^{2}+\frac{599}{27000} \gamma^{3}+\frac{1}{144} \gamma^{2} \alpha_{1} \alpha_{2}\right) \eta^{5} & +\left(\frac{1}{280} \gamma^{2} \alpha_{3} \alpha_{1}^{2}+\frac{1424}{7875} \gamma^{3} \alpha_{1}+\frac{1961}{22500} \gamma^{3} \alpha_{3}\right) \eta^{7} \\
+\left(\frac{989}{147000} \alpha_{1} \gamma^{3}+\frac{2533}{63000} \gamma^{3} \alpha_{2}+\frac{1}{3920} \gamma^{2} \alpha_{2} \alpha_{1}^{2}\right) \eta^{6} & +\cdots, & \phi^{\prime}(\eta)=\alpha_{3}+\frac{8}{5} \gamma \eta+\left(\frac{13}{45} \gamma \alpha_{3}+\frac{2}{9} \alpha_{1} \gamma\right) \eta^{2} \\
+ & +, & +\left(\frac{23}{60} \gamma^{2}+\frac{1}{16} \gamma \alpha_{1} \alpha_{3}\right) \eta^{3}
\end{array}
$$




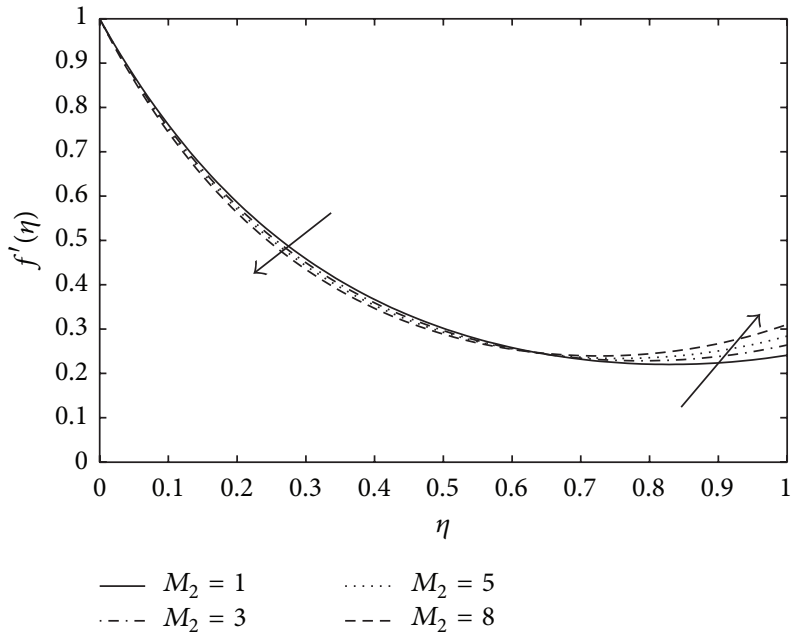

(a)

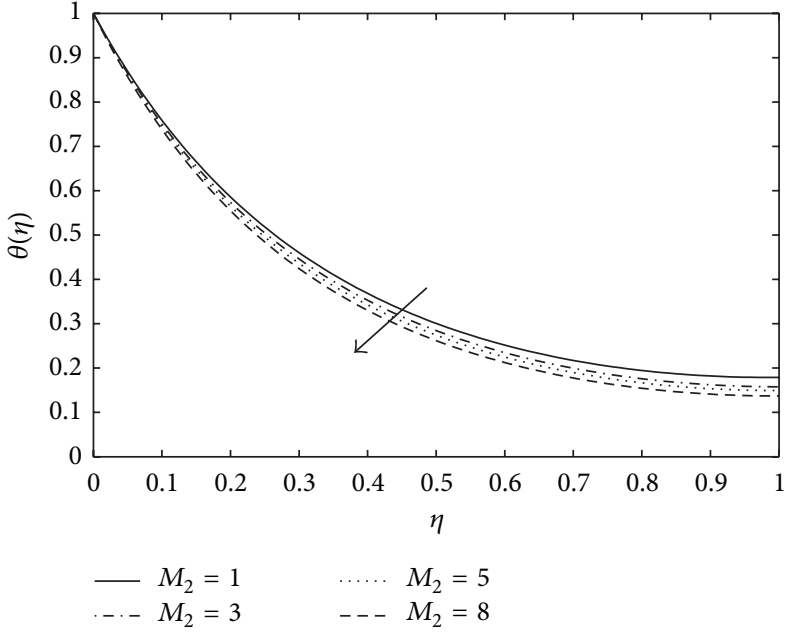

(b)

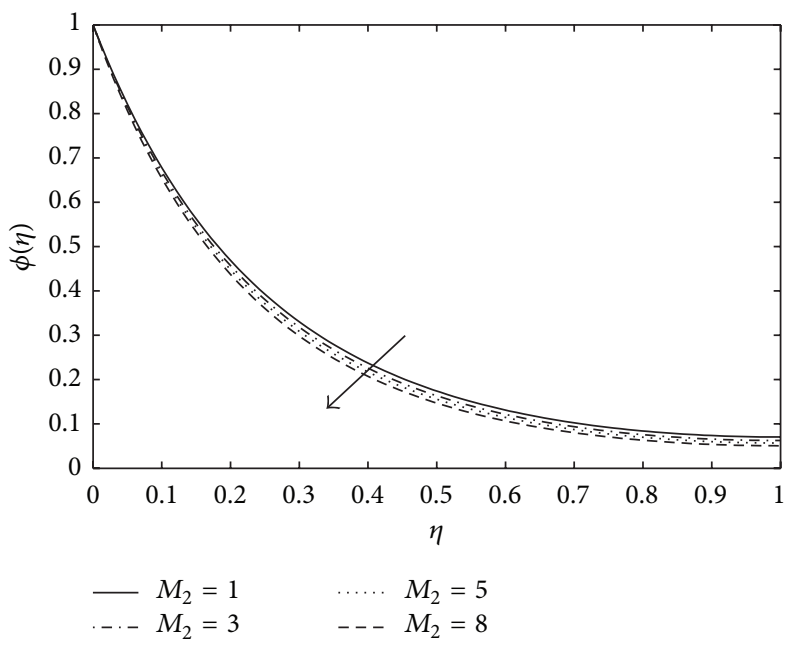

(c)

Figure 5: (a) Velocity profiles for different values of $M_{2}$ with $S=0.8, \mathrm{Ma}=1, M_{1}=1, \operatorname{Pr}=1$, and $\mathrm{Sc}=1.8$. (b) Temperature profiles for different values of $M_{2}$ with $S=0.8, \mathrm{Ma}=1, M_{1}=1, \operatorname{Pr}=1$, and $\mathrm{Sc}=1.8$. (c) Concentration profiles for different values of $M_{2}$ with $S=0.8$, $\mathrm{Ma}=1, M_{1}=1, \operatorname{Pr}=1$, and $\mathrm{Sc}=1.8$.

$$
\begin{aligned}
& +\left(\frac{133}{1875} \gamma^{2} \alpha_{3}+\frac{13}{125} \gamma^{2} \alpha_{1}\right) \eta^{4} \\
& +\left(\frac{1171}{13500} \gamma^{3}+\frac{1}{216} \gamma^{2} \alpha_{1}^{2}+\frac{47}{2700} \gamma^{2} \alpha_{1} \alpha_{3}\right) \eta^{5} \\
& +\left(\frac{1}{1960} \gamma^{2} \alpha_{3} \alpha_{1}^{2}+\frac{1424}{22125} \gamma^{3} \alpha_{1}+\frac{1961}{157500} \gamma^{3} \alpha_{3}\right) \eta^{6} \\
& +\cdots
\end{aligned}
$$

Substituting (21) into (12), we can get four equations involving the four variables such as $\alpha_{1}, \alpha_{2}, \alpha_{3}$, and $\gamma$. Analytical solutions of (10)-(12) in the form of series can be obtained with this method.

\section{Results and Discussion}

The effects of physical parameters on velocity, temperature, and concentration fields are presented in Tables 1-7, respectively. Based on Table 1, the values of dimensionless film thickness $\beta$ and skin friction coefficient $-f^{\prime \prime}(0)$ obtained are compared with the results by Wang [19] for the case $\mathrm{Ma}=0$, and good agreement can be seen. From Table 2, it can be observed that increasing the value of unsteadiness parameter $S$ will decrease the film thickness $\beta$, the skin friction coefficient $-f^{\prime \prime}(0)$, the heat flux $-\theta^{\prime}(0)$, and the diffusion flux $-\phi^{\prime}(0)$ but increase the free velocity $f^{\prime}(1)$, free temperature $\theta(1)$, and free concentration $\phi(1)$. In Table 3, as the magnetic parameter Ma increases, the hydrodynamics behavior is the same as that in Table 2 except for the skin friction coefficient $-f^{\prime \prime}(0)$. 


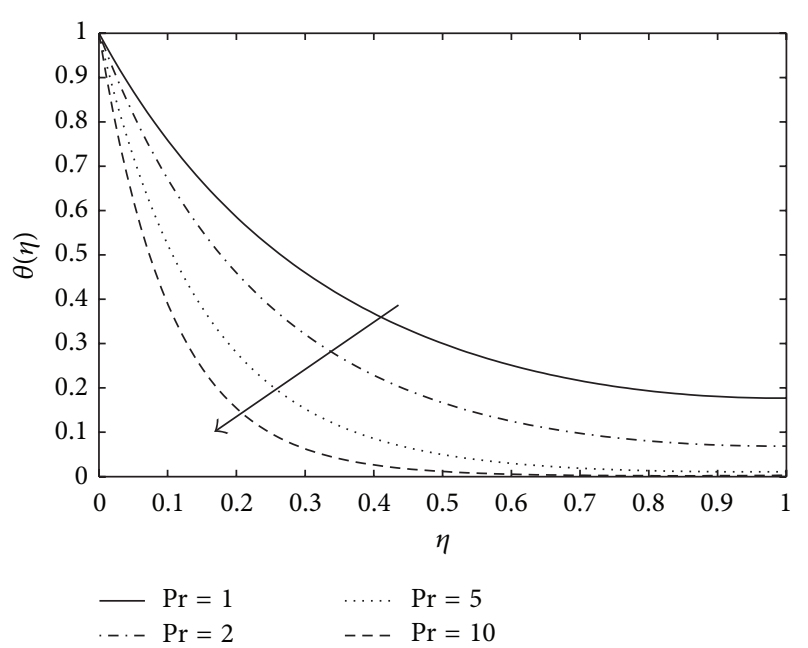

Figure 6: Temperature profiles for different values of $\operatorname{Pr}$ with $S=$ $0.8, \mathrm{Ma}=1, M_{1}=1, M_{2}=1$, and $\mathrm{Sc}=1.8$.

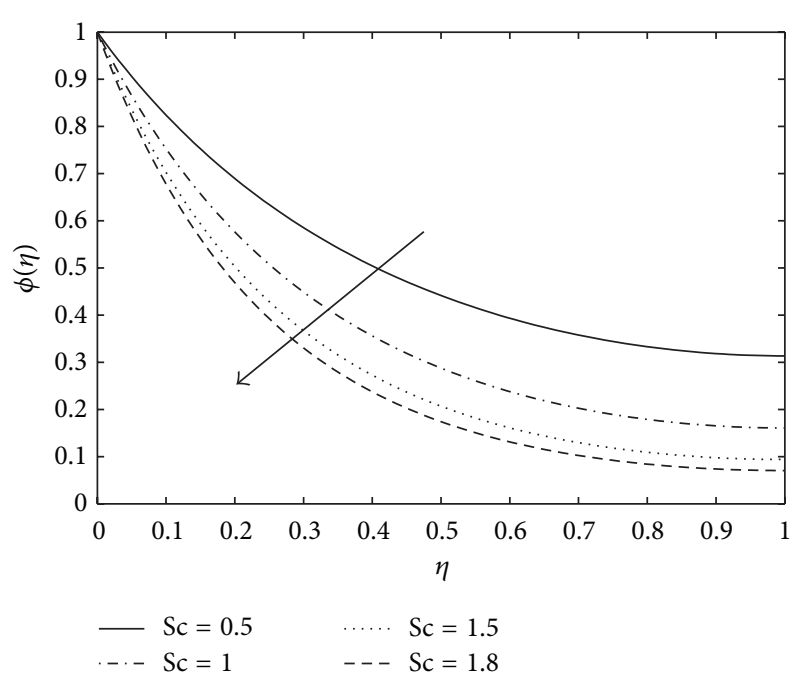

FIGURE 7: Concentration profiles for different values of Sc with $S=$ $0.8, \mathrm{Ma}=1, M_{1}=1, M_{2}=1$, and $\operatorname{Pr}=1$.

From Tables 4 and 5 , it can be seen that $M_{1}$ and $M_{2}$ have the same effects on velocity, temperature, and concentration fields. Increasing the value of the thermocapillary number $M_{1}$ or solutal capillary number $M_{2}$ can cause a descent in the free temperature $\theta(1)$ and free concentration $\phi(1)$ but a rise in the film thickness $\beta$, the skin friction coefficient $-f^{\prime \prime}(0)$, the heat flux $-\theta^{\prime}(0)$, and the free velocity $f^{\prime}(1)$. Table 6 shows that the values of the free concentration $\phi(1)$ and the heat flux $-\theta^{\prime}(0)$ increase while the film thickness $\beta$, the skin friction coefficient $-f^{\prime \prime}(0)$, the diffusion flux $-\phi^{\prime}(0)$, the free velocity $f^{\prime}(0)$, and free temperature $\theta(1)$ decrease with an increment in the Prandtl number Pr. Table 7 shows the effect of Schmidt number Sc on velocity, temperature, and concentration fields.

The velocity, temperature, and concentration profiles for the hydromagnetic flow in a thin film over an unsteady stretching sheet with thermocapillary are presented graphically in Figures 2-7. Figures 2(a)-2(c) show the impacts of unsteadiness parameter on the velocity, temperature, and concentration, respectively. We can find the enhancement in the velocity is more remarkable compared with the temperature and concentration when increment of $S$ is the same. And unsteadiness parameter has the similar effects on the temperature and concentration fields. Figures 3(a)-3(c) show the effects of Hartmann number on the velocity, temperature, and concentration fields, which are the same as Table 3. From Figure 3(a), it can be seen that when the Hartmann number Ma increases, at the beginning, the velocity decreases slightly due to the fact that magnetic field produces a drag, and then the velocity near the surface of the thin film rises up gradually under the Marangoni effect, which explains the emergence of the intersection. Meanwhile, the temperature and concentration rise significantly and monotonously as Ma increases (see Figures 3(b) and 3(c)). It can be proved that the magnetic field promotes heat and mass transfer in a thin liquid film.

Figures 4(a) and 5(a) show the velocity profiles for different values of thermocapillary number $M_{1}$ and solutal capillary number $M_{2}$. Similar to Figure 3(a), with increasing value of thermocapillary number or solutal capillary number, one can see that the velocity decreases initially mainly due to the magnetic field effect; then the velocity near the surface of the thin film obviously rises up mainly because of the Marangoni effect; furthermore, the two factors are balanced at the intersection. The effects of thermocapillary number $M_{1}$ on the temperature and concentration field are shown in Figures 4(b) and 4(c). Results demonstrate that the temperature and concentration both reduce when the thermocapillary number increases. Figures 5(b) and 5(c) illustrate that the fluid temperature and concentration are the decreasing functions of solutal capillary number. The lower value of thermocapillary number has higher temperature and concentration. Furthermore, decrement in the temperature and concentration profiles due to thermocapillary number $M_{1}$ is more remarkable in comparison to the solutal capillary number $M_{2}$.

The effect of Pr on temperature is presented in Figure 6. It can be observed that heat transfer behaviors are strongly dependent on the value of the Prandtl number. Figure 6 shows that the temperature of the fluid decreases monotonously with the increasing of Pr. That is to say, Pr plays an opposite effect on the temperature field compared with $S$ and Ma. In addition, the effect of Schmidt number on the concentration distribution is displayed in Figure 7. Increasing the Schmidt number causes a decrease in the concentration profile, and it is clear that weaker mass transfer ratio is obtained with bigger Sc values.

\section{Conclusions}

In this study, a similarity analysis for thermosolutal capillarity and a magnetic field in a thin liquid film on an unsteady elastic stretching sheet has been studied. The effects of various relevant parameters on the velocity, temperature, and concentration fields have been discussed. The main points of presented analysis are listed below:

(1) The thermocapillary number and solutal capillary number both restrict the heat and mass transfer. 
Furthermore, the effects of the thermocapillary number on the temperature and concentration are more remarkable than solutal capillary number.

(2) The velocity, temperature, and concentration all increase for the higher values of unsteadiness parameter.

(3) Increasing Prandtl number can reduce the fluid temperature, and increasing Schmidt number causes a descent in the concentration.

(4) Marangoni convection caused by surface-tension gradient creates a promotion in flow near the surface region of the film.

\section{Nomenclature}

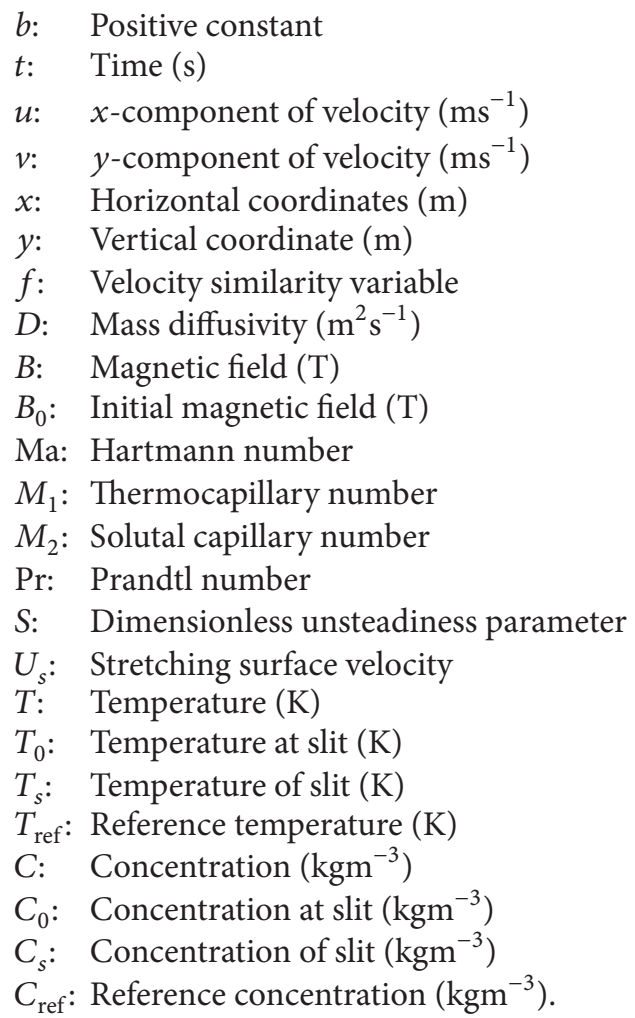

\section{Greek Symbols}

$\begin{array}{ll}\alpha, \alpha_{1}, \alpha_{2}, \alpha_{3}: & \text { Constants } \\ \beta: & \text { Dimensionless film thickness } \\ \kappa: & \text { Thermal diffusivity }\left(\mathrm{m}^{2} \mathrm{~s}^{-1}\right) \\ \widehat{\sigma}: & \text { Electrical conductivity }\left(\mathrm{s}(\mathrm{cm})^{-1}\right) \\ \eta: & \text { Similarity variable } \\ \mu: & \text { Dynamic viscosity }\left(\mathrm{Nsm}^{-2}\right) \\ v: & \text { Kinematic viscosity }\left(\mathrm{m}^{2} \mathrm{~s}^{-1}\right) \\ \gamma: & \text { Dimensionless film thickness } \\ \theta: & \text { Temperature similarity variable } \\ \phi: & \text { Concentration similarity variable } \\ \varepsilon: & \text { Artificial small parameter } \\ \rho: & \text { Density }\left(\mathrm{kgm}{ }^{-3}\right) \\ \sigma: & \text { Surface tension }\left(\mathrm{Nm}^{-1}\right) .\end{array}$

\section{Subscripts}
T: Thermal quantity
$C$ : Solutal quantity
0 : At the slit
$s$ : At the elastic sheet.

\section{Competing Interests}

The authors declare that there is no conflict of interests regarding the publication of this paper.

\section{Acknowledgments}

This work is supported by the National Natural Science Foundation of China (nos. 21206009 and 21576023) and Funding Project for Academic Human Resources Development in Beijing University of Civil Engineering and Architecture (no. 21221214111)

\section{References}

[1] L. J. Crane, "Flow past a stretching plate," Zeitschrift für angewandte Mathematik und Physik ZAMP, vol. 21, no. 4, pp. 645-647, 1970.

[2] S. Munawar, A. Mehmood, and A. Ali, "Time-dependent flow and heat transfer over a stretching cylinder," Chinese Journal of Physics, vol. 50, no. 5, pp. 828-848, 2012.

[3] S. A. Shehzad, Z. Abdullah, A. Alsaedi, F. M. Abbasi, and T. Hayat, "Thermally radiative three-dimensional flow of Jeffrey nanofluid with internal heat generation and magnetic field," Journal of Magnetism \& Magnetic Materials, vol. 397, pp. 108$114,2016$.

[4] C. Y. Wang, "The three-dimensional flow due to a stretching flat surface," The Physics of Fluids, vol. 27, no. 8, pp. 1915-1917, 1984.

[5] S. Munawar, A. Mehmood, and A. Ali, "Three-dimensional squeezing flow in a rotating channel of lower stretching porous wall," Computers \& Mathematics with Applications, vol. 64, no. 6, pp. 1575-1586, 2012.

[6] M. E. Ali and E. Magyari, "Unsteady fluid and heat flow induced by a submerged stretching surface while its steady motion is slowed down gradually," International Journal of Heat and Mass Transfer, vol. 50, no. 1-2, pp. 188-195, 2007.

[7] M. M. Rashidi, M. Ali, B. Rostami, P. Rostami, and G.-N. Xie, "Heat and mass transfer for MHD viscoelastic fluid flow over a vertical stretching sheet with considering Soret and Dufour effects," Mathematical Problems in Engineering, vol. 2015, Article ID 861065, 12 pages, 2015.

[8] P. V. S. Narayana and D. H. Babu, "Numerical study of MHD heat and mass transfer of a Jeffrey fluid over a stretching sheet with chemical reaction and thermal radiation," Journal of the Taiwan Institute of Chemical Engineers, vol. 59, pp. 18-25, 2016.

[9] A. Mehmood, S. Munawar, and A. Ali, "Cooling of a hot stretching surface in the presence of across mass transfer phenomenon in a channel flow," Zeitschrift fur Naturforschung A, vol. 69, no. 1-2, pp. 34-42, 2014.

[10] S. A. Shehzad, T. Hayat, and A. Alsaedi, "Three-dimensional MHD flow of Casson fluid in porous medium with heat 
generation," Journal of Applied Fluid Mechanics, vol. 9, no. 1, pp. 215-223, 2016.

[11] Y. Zhang and L. C. Zheng, "Analysis of MHD thermosolutal Marangoni convection with the heat generation and a firstorder chemical reaction," Chemical Engineering Science, vol. 69, no. 1, pp. 449-455, 2012.

[12] T. Hayat, A. Safdar, M. Awais, and S. Mesloub, "Soret and Dufour effects for three-dimensional flow in a viscoelastic fluid over a stretching surface," International Journal of Heat and Mass Transfer, vol. 55, no. 7-8, pp. 2129-2136, 2012.

[13] S. A. Shehzad, Z. Abdullah, F. M. Abbasi, T. Hayat, and A. Alsaedi, "Magnetic field effect in three-dimensional flow of an Oldroyd-B nanofluid over a radiative surface," Journal of Magnetism \& Magnetic Materials, vol. 399, pp. 97-108, 2016.

[14] A. Mastroberardino, "Accurate solutions for viscoelastic boundary layer flow and heat transfer over stretching sheet," Applied Mathematics and Mechanics, vol. 35, no. 2, pp. 133-142, 2014.

[15] J. A. Khan, M. Mustafa, T. Hayat, and A. Alsaedi, "Threedimensional flow of nanofluid over a non-linearly stretching sheet: an application to solar energy," International Journal of Heat and Mass Transfer, vol. 86, pp. 158-164, 2015.

[16] S. A. Shehzad, F. M. Abbasi, T. Hayat, F. Alsaadi, and G. Mousa, "Peristalsis in a curved channel with slip condition and radial magnetic field," International Journal of Heat and Mass Transfer, vol. 91, pp. 562-569, 2015.

[17] C. Y. Wang, "Liquid film on an unsteady stretching surface," Quarterly of Applied Mathematics, vol. 48, no. 4, pp. 601-610, 1990.

[18] S. M. Roberts and J. S. Shipman, Two Point Boundary Value Problems: Shooting Methods, Elsevier, New York, NY, USA, 1972.

[19] C. Wang, "Analytic solutions for a liquid film on an unsteady stretching surface," Heat and Mass Transfer, vol. 42, no. 8, pp. 759-766, 2006.

[20] M. M. Nandeppanavar, K. Vajravelu, M. Subhas Abel, S. Ravi, and H. Jyoti, "Heat transfer in a liquid film over an unsteady stretching sheet," International Journal of Heat and Mass Transfer, vol. 55, no. 4, pp. 1316-1324, 2012.

[21] Y. Zhang, M. Zhang, and Y. Bai, "Flow and heat transfer of an Oldroyd-B nanofluid thin film over an unsteady stretching sheet," Journal of Molecular Liquids, vol. 220, pp. 665-670, 2016.

[22] H. I. Andersson, J. B. Aarseth, and B. S. Dandapat, "Heat transfer in a liquid film on an unsteady stretching surface," International Journal of Heat and Mass Transfer, vol. 43, no. 1, pp. 69-74, 2000.

[23] C.-H. Chen, "Heat transfer in a power-law fluid film over a unsteady stretching sheet," Heat and Mass Transfer, vol. 39, no. 8-9, pp. 791-796, 2003.

[24] B. S. Dandapat, B. Santra, and K. Vajravelu, "The effects of variable fluid properties and thermocapillarity on the flow of a thin film on an unsteady stretching sheet," International Journal of Heat and Mass Transfer, vol. 50, no. 5-6, pp. 991-996, 2007.

[25] I.-C. Liu and H. I. Andersson, "Heat transfer in a liquid film on an unsteady stretching sheet," International Journal of Thermal Sciences, vol. 47, no. 6, pp. 766-772, 2008.

[26] C. Wang and I. Pop, "Analysis of the flow of a power-law fluid film on an unsteady stretching surface by means of homotopy analysis method," Journal of Non-Newtonian Fluid Mechanics, vol. 138, no. 2-3, pp. 161-172, 2006.
[27] C.-H. Chen, "Effect of viscous dissipation on heat transfer in a non-Newtonian liquid film over an unsteady stretching sheet," Journal of Non-Newtonian Fluid Mechanics, vol. 135, no. 2-3, pp. 128-135, 2006.

[28] N. F. Noor, O. Abdulaziz, and I. Hashim, "MHD flow and heat transfer in a thin liquid film on an unsteady stretching sheet by the homotopy analysis method," International Journal for Numerical Methods in Fluids, vol. 63, no. 3, pp. 357-373, 2010.

[29] M. S. Abel, N. Mahesha, and J. Tawade, "Heat transfer in a liquid film over an unsteady stretching surface with viscous dissipation in presence of external magnetic field," Applied Mathematical Modelling, vol. 33, no. 8, pp. 3430-3441, 2009.

[30] B. S. Dandapat, B. Santra, and H. I. Andersson, "Thermocapillarity in a liquid film on an unsteady stretching surface," International Journal of Heat and Mass Transfer, vol. 46, no. 16, pp. 3009-3015, 2003.

[31] N. F. M. Noor and I. Hashim, "Thermocapillarity and magnetic field effects in a thin liquid film on an unsteady stretching surface," International Journal of Heat and Mass Transfer, vol. 53, no. 9-10, pp. 2044-2051, 2010.

[32] I. Pop, A. Postelnicu, and T. Groşan, “Thermosolutal Marangoni forced convection boundary layers," Meccanica, vol. 36, no. 5, pp. 555-571, 2001.

[33] Y. H. Lin, L. C. Zheng, and X. X. Zhang, "Radiation effects on Marangoni convection flow and heat transfer in pseudo-plastic non-Newtonian nanofluids with variable thermal conductivity," International Journal of Heat and Mass Transfer, vol. 77, no. 4, pp. 708-716, 2014.

[34] Y. H. Lin, L. C. Zheng, X. X. Zhang, L. X. Ma, and G. Chen, "MHD pseudo-plastic nanofluid unsteady flow and heat transfer in a finite thin film over stretching surface with internal heat generation," International Journal of Heat and Mass Transfer, vol. 84, pp. 903-911, 2015.

[35] E. Lim and Y. M. Hung, "Thermophysical phenomena of working fluids of thermocapillary convection in evaporating thin liquid films," International Communications in Heat and Mass Transfer, vol. 66, pp. 203-211, 2015.

[36] D. M. Christopher and B.-X. Wang, "Similarity simulation for Marangoni convection around a vapor bubble during nucleation and growth," International Journal of Heat and Mass Transfer, vol. 44, no. 4, pp. 799-810, 2001.

[37] S. Maity, Y. Ghatani, and B. S. Dandapat, "Thermocapillary flow of a thin nanoliquid film over an unsteady stretching sheet," Journal of Heat Transfer, vol. 138, no. 4, Article ID 042401, 2016.

[38] E. A. Chinnov, "Wave-thermocapillary effects in heated liquid films at high Reynolds numbers," International Journal of Heat and Mass Transfer, vol. 71, no. 1, pp. 106-116, 2014.

[39] H. Schichting, Boundary Layer Theory, McGraw-Hill, New York, NY, USA, 6th edition, 1964.

[40] K. Arafune and A. Hirata, "Thermal and solutal marangoni convection in In-Ga-Sb system," Journal of Crystal Growth, vol. 197, no. 4, pp. 811-817, 1999. 


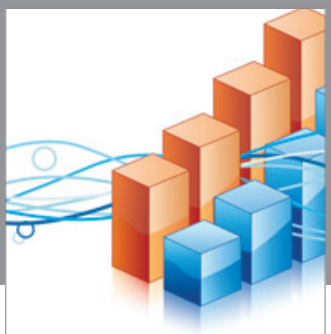

Advances in

Operations Research

vatem alat4

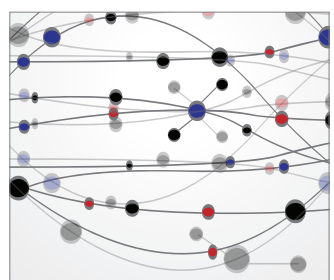

\section{The Scientific} World Journal
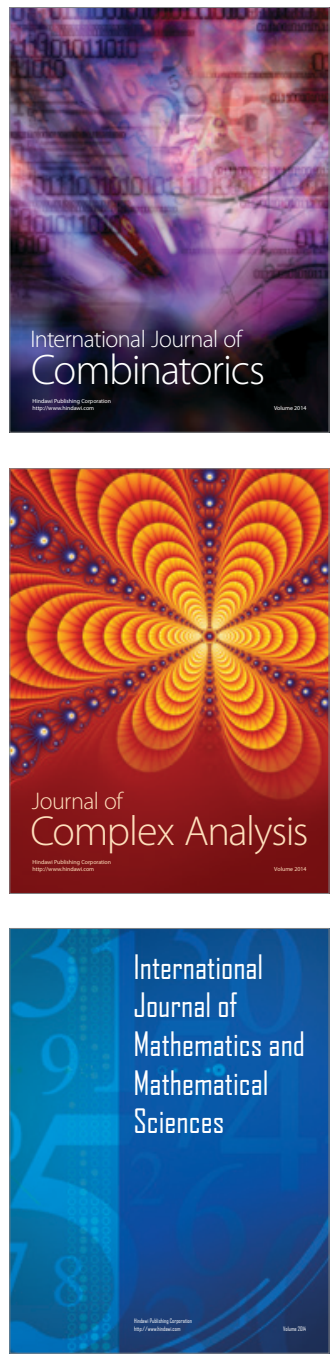
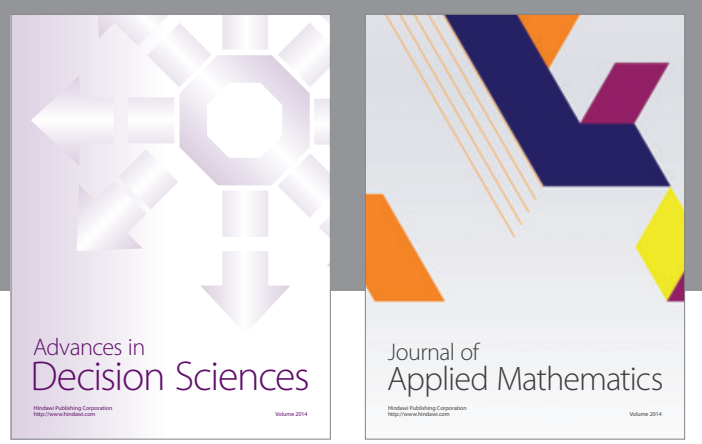

Algebra

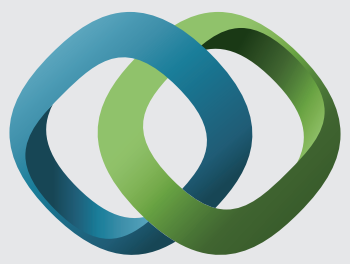

\section{Hindawi}

Submit your manuscripts at

http://www.hindawi.com
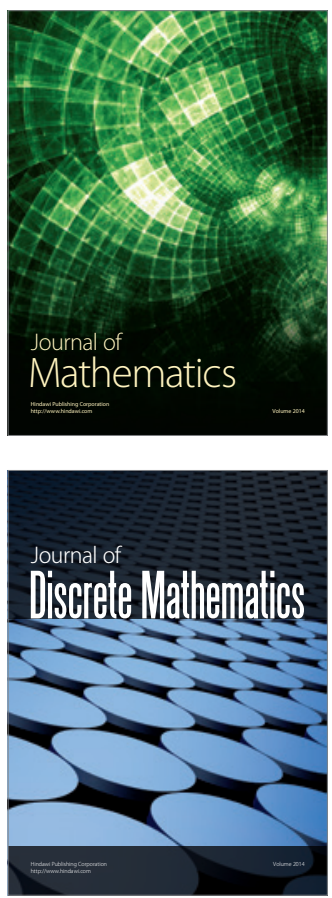

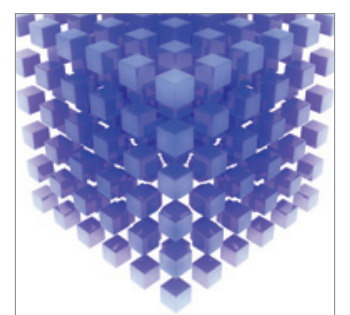

Mathematical Problems in Engineering
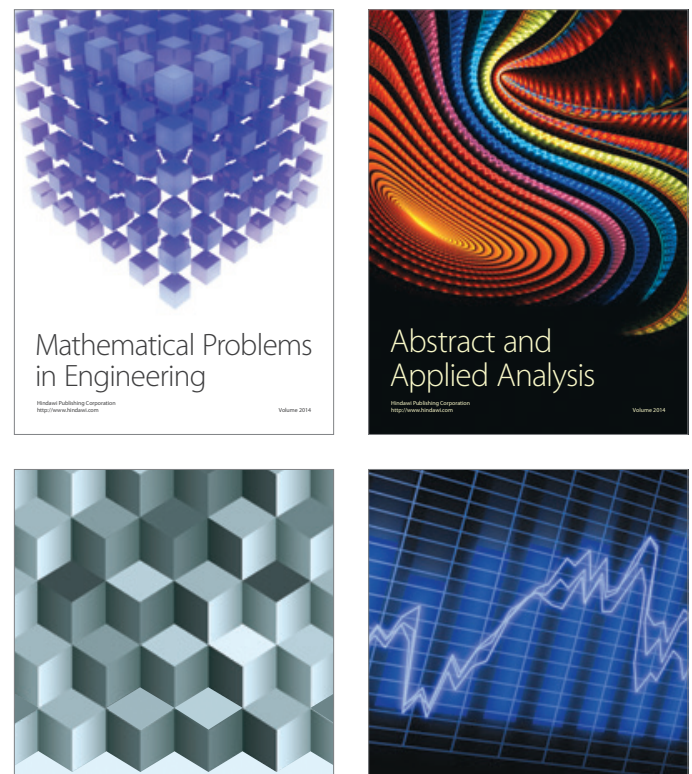

Journal of

Function Spaces

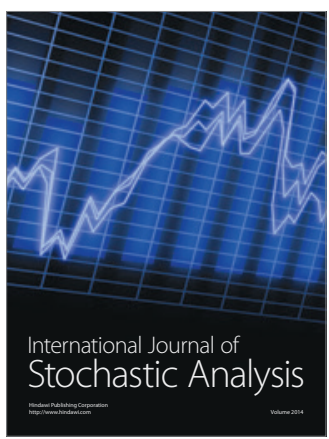

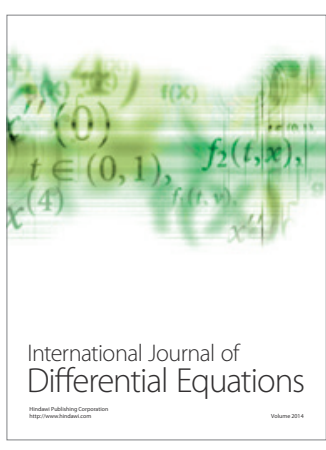
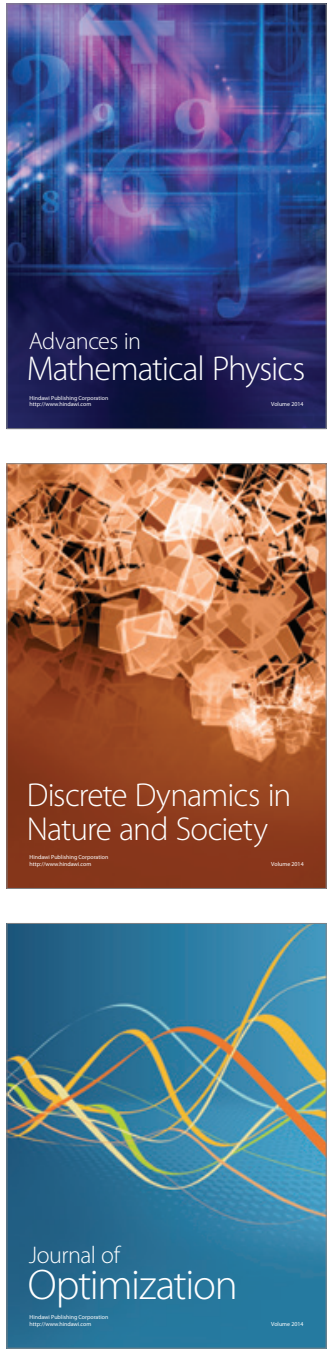